complex (against a picric acid compensator) is plotted at different concentrations of creatinine, not only does $\lambda_{\max }$. vary, but so also does $E$ (1 per cent, $1 \mathrm{~cm}$.).

Most clinical methods recommend that the colour produced in this reaction should be read using a 520-m $\mu$ filter. An explicit reason for this can now be given, for the absorption curves become closely convergent at a wave-length of about $515 \mathrm{~m} \mu$.

The findings indicate that the reaction postulated by Greenwald ${ }^{3}$, in which one molecule of picric acid is said to react with one molecule of creatinine to produce a tautomeric (enolic) form of creatinine picrate, cannot be as simple as considered hitherto. R. J. GARNER

Biochemistry Department, University, Liverpool 3. April 2.

${ }^{1}$ Miller, B. F., and Dubos, R., J. Biol. Chem., 121, 457 (1937).

${ }^{2}$ Peters, J. H., J. Biol. Chem., 146, 179 (1942).

${ }^{8}$ Greenwald, I., J. Amer. Chem. Soc., 47, 1443 (1925).

\section{Isolation of 11 : I2-Benzfluoranthene from a Coal Tar Fraction}

A RECENT description by Kruber and Oberkobusch ${ }^{1}$ of the isolation from a coal tar pitch fraction of an unidentified hydrocarbon $\mathrm{C}_{20} \mathrm{H}_{12}$ (m.p. $216^{\circ} \mathrm{C}$.) prompts me to record the isolation from a corresponding coal tar fraction of a hydrocarbon which possesses similar properties and which has been identified as 11:12-benzfluoranthene (I). The substance was obtained from a fraction (b.p. $269-279^{\circ} \mathrm{C} . / 5 \mathrm{~mm}$.) produced by high-efficiency fractionation of the neutral portion of a distillate obtained during the production of pitch coke. By crystallizing the soft resinous fraction from ether, $\mathbf{b}_{0}$ yellow solid was obtained which was purified by the crystallization and subsequent decomposition of its $1: 3: 5$-trinitrobenzene derivative. The pure hydrocarbon crystallized from benzene as very pale yellow needles, m.p. $216-217^{\circ} \mathrm{C}$. It had a molecular formula of $\mathrm{C}_{20} \mathrm{H}_{12}$, and its ultra-violet absorption spectrum was identical with that published for 11 : 12-benzfluoranthene ${ }^{2}$.

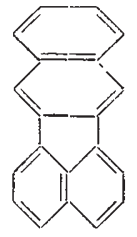

(I)

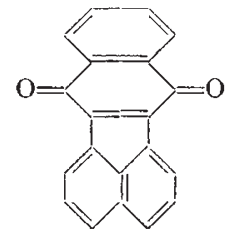

(II)
The identity of the hydrocarbon was confirmed by comparison with a synthetic specimen of $11: 12$ benzfluoranthene prepared according to the method described by Orchin and Reggel ${ }^{2}$. The following derivatives of the coal tar hydrocarbon were also identical with the corresponding complexes formed from the synthetic hydrocarbon: unstable picrate (red needles, m.p. $167-168^{\circ} \mathrm{C}$.), $1: 3: 5$-trinitrobenzene derivative (golden-yellow needles, m.p. 181-182 C.), 2:4: 7-trinitrofluorenone derivative

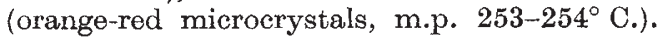

Oxidation of both the synthetic and coal tar hydrocarbons with potassium dichromate in acetic acid yielded a quinone which, after purification by chromatography on activated alumina, crystallized as bright red needles, m.p. $236-237^{\circ} \mathrm{C}$. Analytical figures were in agreement with the formula $\mathrm{C}_{20} \mathrm{H}_{10} \mathrm{O}_{2}$. Further oxidation produced naphthalic anhydride, indicating that the quinone has the structure (II), assigned to it by Moureu, Chovin and Rivoal ${ }^{3}$.

A complete account of the investigation of the pitch-coking distillate will be published at a later date.

Coal Tar Research Association,

A. S. HARRIS Gomersal, Nr. Leeds. July 22.

${ }^{1}$ Kruber, O., and Oberkobusch, R., Chem. Ber., 85, 433 (1952). ${ }^{2}$ Orchin, M., and Reggel, L., J. Amer. Chem. Soc., 73, 436 (1951). ${ }^{3}$ Moureu, H., Chovin, P., and Rivoal, G., Bull. Soc. Chim., France, 99 (1948).

\section{Sugar Components of Blood and Urinary Glycoproteins: Confirmation of the Presence of Fucose by a New Reaction}

DURING the course of a systematic study of the carbohydrate constituents of some glycoproteins of human and animal origin, a number of unexpected component sugars have been separated by paper chromatography. In particular, the presence of fucose has been definitely demonstrated in human serum mucoprotein and in normal human urinary proteins.

Serum mucoprotein was prepared by the method of Weumer et al. ${ }^{1}$. To prepare the urinary protein, normal urine was dialysed and concentrated to onefifth of its original volume at room temperature and reduced pressure. Six volumes of alcohol were added to precipitate the proteins. An examination of this material by paper strip electrophoresis showed the presence of albumin, $\alpha_{1}, \alpha_{2}$ and $\beta$ globulins, these latter in rather low concentration.

The glycoproteins were hydrolysed with $2 \mathrm{~N}$ sulphuric acid for $8 \mathrm{hr}$. at $100^{\circ} \mathrm{C}$. in sealed tubes. The hydrolysates were neutralized to $p \mathrm{H} 4.5$ with barium hydroxide and the clear filtrate concentrated to a suitable volume. Aliquots were then chromatographed using pyridine/amyl alcohol/water, phenol/ water for 3 per cent ammonia, or butanol/ethanol/water as solvent systems. The chromatograms were sprayed with $p$-anisidine hydrochloride in butanol ${ }^{2}$. Mannose, galactose, and a hexosamine were found, corresponding to the results obtained by Werner and Odin ${ }^{3}$ for ox seromucoid. The hexosamine was identified as glucosamine $e^{4}$ and some of it is present in the native protein as the $\mathrm{N}$-acetylated derivative. A faster moving component was invariably present on the chromatograms, however. It was found to have an $R_{F}$ value corresponding to that of fucose in all the solvent systems used. The intensity of the spot relative to the other sugar components was greater in the urinary material than in the serum mucoprotein.

In order to confirm the identity of this sugar, advantage was taken of the fact that periodate oxidizes methylpentoses to formic acid and acetaldehyde, whereas simple aldoses and ketoses are oxidized to formic acid and formaldehyde ${ }^{5}$. Acetaldehyde can be readily detected by the Rimini reaction ${ }^{6}$ with piperazine and nitroprusside, the full scope and limitations of which will be described elsewhere ${ }^{7}$. This test can be applied to paper chromatograms, which are sprayed with $2 \cdot 5$ per cent aqueous sodium metaperiodate. The oxidation is allowed to proceed for ten minutes and then the Rimini reagent is applied. This reagent must be prepared immediately before use by mixing 7 per cent aqueous sodium nitro- 